\title{
La capture sacrificielle du pangolin en Afrique centrale
}

The sacrificial capture of the pangolin in central Africa

Luc de Heusch

\section{(2) OpenEdition}

\section{Journals}

Édition électronique

URL : http://journals.openedition.org/span/541

DOI : 10.4000/span.541

ISSN : 2268-1558

\section{Éditeur}

École pratique des hautes études. Sciences humaines

\section{Édition imprimée}

Date de publication : 1 avril 1984

Pagination : 131-147

ISSN : 0294-7080

\section{Référence électronique}

Luc de Heusch, "La capture sacrificielle du pangolin en Afrique centrale ", Systèmes de pensée en Afrique noire [En ligne], 6 | 1984, mis en ligne le 05 juin 2013, consulté le 01 mai 2019. URL : http:// journals.openedition.org/span/541; DOI : 10.4000/span.541 
LA C A P T U R E S A C R I F I C I E L L E



par

Luc de Heusch

Animaux-esprits et animaux dégoûtants

Une société bantoue du Zaĩre se distinguait radicalement de toutes les autres jusqu'à une époque récente par la quasi absence de pratique sacrificielle : les Lele du Kasaī. Les brillants travaux de Mary Douglas ont rendu célèbres ces agriculteurs-chasseurs qui manifestent une complète absence d'intérêt pour toute espèce d'élevage, à l'exception de celui du chien. Nous nous trouvons ici en présence d'un paradoxe. Toutes les relations rituelles des hommes avec les esprits de la nature (mingehe) se font par l'intermédiaire des animaux sauvages. Les esprits habitent les profondeurs des forêts, spécialement les sources. "Ils contrôlent la fertilité des femmes et la prospérité de la chasse masculine. Ils peuvent aussi retenir le gibier et faire dévier la flèche du chasseur" (Douglas M., 1954 : 10). Le problème du statut des animaux sauvages a été fort bien posé en termes symboliques par Mary Douglas : "Certains animaux et certaines plantes témoignent par des signes, écrit-t-elle, qu'ils sont associés à des esprits de manière particulièrement étroite" (Ibia.). Ce système symbolique permet de comprendre aussi bien les interdits rituels des Lele que leurs rites positifs, dont la fonction évidente est de maintenir de bonnes relations entre le village, domaine des hommes, et la forêt, domaine des esprits. Tentons de systématiser quelque peu les informations de

(*) Cet article est extrait d'un ouvrage sous presse en anglais, intitulé Sacrifice in Africa (Manchester University Press). 
Mary Douglas, dispersées dans plusieurs études.

Les esprits-animaux sont en premier lieu les créatures aquatiques. D'une manière générale, les femmes (ou seulement les femmes enceintes) doivent les éviter (Douglas 1957 : 48-49). On citera en premier 1ieu les poissons. Ceux-ci sont toujours 1'objet d'un traitement ritue1 avant d'être introduits au village (Douglas 1954: 10 ; 1957 : 52). Les mammifères terrestres qui manifestent une prédilection pour l'eau sont également associés aux esprits. Il en est ainsi du cochon sauvage qui passe son temps à se vautrer dans les sources. Les Lele le considèrent comme le chien des esprits : il vit et mange avec eux, leur obéīt (Douglas 1954 : $10 ; 1957$ : 49). On situera dans la même catégorie le chevrotain d'eau (hyemoschus aquaticus) "qui se cache en plongeant dans I'eau jusqu'aux narines" (Douglas 1957 : 49), ainsi que le babouin jaune qui aime se laver, à 1 'inverse des autres singes qui évitent 1'eau (Douglas 1975 : 301).

Bien que ce ne soit pas exactement le point de vue adopté par Mary Douglas, 1 'on pourrait dire que ces animaux sont associés aux esprits de l'eau par une relation métonymique de contiguïté. Le même type de lien symbolique existe entre les esprits des morts et certaines espèces creusant un terrier (Douglas 1975 : 301 ; 1957 : 49).

Les esprits de l'eau se manifestent la nuit. A ce titre une espèce leur est associée en vertu d'une relation métaphorique. I1 s'agit de 1 'antilope cephatophus grimmi qui sommeille durant la journée, les yeux grands ouverts (Douglas 1957 : 49).

Métaphore et métonymie n'épuisent pas cependant le projet classificatoire des Lele. Une frontière décisive sépare théoriquement le village, domaine des hommes, et la forêt, domaine des animaux et des esprits. Toutes les espèces qui se manifestent au village et aux alentours sont dès lors anomaliques, suspectes. C'est la raison pour laquelle les Lele n'élèvaient ni chèvres ni porcs avant une époque récente. Les seuls animaux tolérés étaient le chien et la poule, bien que leur présence au village fasse problème. Théoriquement tous les animaux domestiques sont impropres à la consommation, ils soulèvent le dégoût, ils sont qualifiés de hama. Cette réaction est plus vive encore envers les rats qui infestent les huttes (Douglas 1957 : 47).

Le concept hama n'a rien à voir avec les interdits de nature religieuse marquant les animaux-esprits. Il s'enracine dans 1 'expérience 
de la saleté corporelle, il est de l'ordre de la "souillure". Il évoque la nausée que soulève la vue du cadavre, des excréments, de la vermine, etc. (Douglas 1955 : $388 ; 1954$ : 5). Les animaux puants comme le chacal participent de la même expérience sensible. Mais le sens premier du terme hama connaît une extension intellectuelle remarquable ; certaines espèces sont qualifiées de hama, dégoûtantes, et ne sont jamais consommées parce qu'elles ne sont pas à leur place dans la topologie et la taxinomie lele. Ces animaux présentent dès lors des caractères "impropres" au sens figuré. C'est le cas non seulement des chiens et des chèvres -- dont la présence est déplacée parmi les hommes -- mais encore de l'ensemble des carnivores. Ceux-ci rôdent à proximité du village, ils transgressent les frontières du monde animal et du monde humain, comme les sorciers dont ils sont les familiers (Douglas 1955 : 391 et 395 ; 1975 : 301).

On notera immédiatement que le sentiment de répulsion envers ces animaux est sujet à variations. Seuls les membres de l'association cultuelle des Géniteurs (begetters) réunissant les hommes qui ont engendré, peuvent consommer impunément la chair des carnassiers. En revanche, tous les hommes consomment volontiers les poulets, seules les femmes s'en abstiennent. Celles-ci considèrent aussi comme hama un animal au statut ambigu, mi-mammifère, mi-oiseau : 1'écureuil volant (Douglas 1955 : $392 ; 1957: 48$ ).

Un nouvel aspect de la topologie symbolique apparâ̂t ici. Les Lele assignent des caractéristiques propres aux animaux qui peuplent respectivement 1'eau, la terre, les airs. Créatures terrestres, les mamifères se meuvent au moyen de quatre pattes; les oiseaux ont des ailes pour voler (Douglas 1957 : 48). L'écureuil volant est donc anomalique. Son comportement est littéralement "impropre", hama. Mary Douglas n'explique guère les raisons pour lesquelles les serpents, les grenouilles et les crapauds se trouvent dans une situation analogue (Douglas 1955 : 388 et 390 ). Compte tenu de la remarque précédente, on constatera cependant que ces créatures hama ne répondent ni à la définition des créatures terrestres (mammifères), ni à celle des poissons, ni à celle des oiseaux. On pourrait dire qu'ils souffrent d'une monstruosité absolue : ils ne sont de nulle part.

Mais le problème se complique car certaines espèces jugées aberrantes du point de vue taxinomique, loin d'être qualifiées de hama, 
entrent dans la catégorie des animaux-esprits. J'ai dialogué jadis à ce propos avec Mary Douglas (de Heusch 1971). J'ai attiré l'attention sur la nécessité de distinguer soigneusement les concepts d'interdit et de souizlure, bien qu'ils puissent se confondre à la limite. Nous avons déjà vu que Evans-Pritchard les a amalgamés bien à tort chez les Nuer. Les animaux-esprits, interdits aux femmes pour des raisons religieuses (car les uns et les autres sont en rapport avec la fécondité) ne sont en rien dégoûtants ou impurs. Ils sont chargés de pouvoir spirituel et l'interdiction de les consommer varie selon le statut personnel. "Certains animaux-esprits sont considérés comme une nourriture dangereuse pour tous, à l'exception des devins; d'autres espèces sont dangereuses pour les femmes enceintes et tous ceux qui subissent un traitement contre la stérilité; d'autres encore sont interdites au cours de rites curatifs spécifiques" (Douglas 1975 : 301). Les femmes sont aussi plus sensibles que les hommes aux animaux hama, mais cette attitude n'a rien à voir avec 1 'évitement qui les détourne du contact ou de la consommation des animaux-esprits. Il $n$ 'en subsiste pas moins une équivoque. Certains comportements animaux insolites sont valorisés, alors que d'autres (celui des carnassiers ou de l'écureuil volant par exemple) sont des attributs hama. Le choix est clair lorsqu'il s'agit des mammifères terrestres qui manifestent une propension pour l'eau, domaine des esprits (comme le cochon sauvage, le chevrotain ou le babouin) (Douglas 1975 : 301). Mais d'autres espèces sịngulières entrent dans la catégorie des animaux-esprits pour des raisons plus obscures. Il n'est pas aisé de comprendre, par exemple, pourquoi la tortue, que les femmes s'abstiennent de consommer, est classée parmi les animauxesprits et non parmi les animaux hama: "elle se distingue des autres reptiles par.sa carapace, mais en tant que créature munie de quatre pattes, son mode de reproduction ovipare est anomalique" (Douglas 1957 : 48).

Les Lele semblent tracer une frontière incertaine entre animauxesprits et animaux dégoûtants. Prenons le cas du varan d'eau (varanus nizoticus). Cet amphibien a un statut ambigu. Dans un premier essai, Mary Douglas nous le présente comme hama : les Lele sont révoltés à 1'idée de manger une créature ressemblant à un serpent (Douglas 1955 : 390). Mais ailleurs, le même animal est classé parmi les animauxesprits. Les femmes ne peuvent même pas toucher le varan d'eau parce 
qu'il diffère des serpents comme des espèces voisines : "Les Lele le décrivent comme un cousin du crocodile, mais dépourvu d'écailles; semblable à un serpent muni de petites pattes ; semblable au lézard, mais plus gros, plus rapide et plus méchant. Comme le crocodile, le varan est un gros amphibien, potentiellement dangereux" (Douglas 1957 : 48).

Or, si l'on classe les animaux-esprits en fonction de leur caractère mystique, le varan d'eau occupe une position privilégiée comparable à celle du petit pangolin, l'animal le plus fortement valorisé. En effet les femmes doivent s'abstenir de tout contact avec ces deux espèces, alors qu'elles peuvent toucher, mais non manger, la tortue et le babouin et qu'elles évitent les espèces aquatiques seulement durant leur grossesse (Douglas 1957 : 48-49).

Le culte du pangolin

Le petit pangolin occupe le centre même de la vie rituelle lele, en tant. que monstre taxinomique. Cette fois il n'y a aucune hésitation sur son statut : nous sommes bien en présence de l'animal-esprit par excellence, et non d'une créature dégoûtante (hama). Pourquoi ? Le petit pangolin transcende toutes les catégories. "Dans nos forêts, disent les Lele, il y a un animal qui a le corps et la queue d'un poisson, couvert d'écailles. Il a quatre pattes, et il grimpe dans les arbres." (Douglas 1957 : 50). Le pangolin se trouve donc associé à l'eau, principe de fertilité, comme le varan (animal amphibie). Mais sa puissance symbolique est infiniment plus grande car ce mammifèrepoisson est un habitant des arbres comme les oiseaux. En outre il présente un caractère humain remarquable : $i 1$ ne met au monde qu'un petit à la fois, contrairement aux autres espèces animales (Douglas 1957 : 50 ; 1975 : 302). Le petit pangolin est un véritable résumé de 1 'univers. I1 cumule les propriétés des créatures aquatiques, célestes et terrestres. Monopare, il est aussi le représentant symbolique de la reproduction humaine bien tempérée dans un univers où la fécondité est innombrable, démesurée. Il est l'opérateur logique -- ou plutôt dialectique -- de la communication religieuse. Par sa médiation, le village et la forêt, les hommes et les esprits entrent en relation de manière privilégiée. 
I1 existe un écart maximal entre le léopard (animal hama comme tous les carnivores) et le petit pangolin, le plus important des animaux-esprits (Douglas 1975 : 302). Le premier est un sorcier, le second est 1 'ami de $I^{\prime}$ homme, et même son représentant métaphorique au sein du monde animal. Il est appelé "chef" (Kum) parce qu'il favorise 1a fécondité des femmes (Douglas 1957 : 55). Il est le seu1 anima1 sensible à la honte (buhonyi); ne baisse-t-il pas la tête comme un homme qui évite de regarder sa belle-mère ? (Douglas 1975 : 302). S'il se laisse capturer par les chasseurs, c'est parce qu'il le veut bien. Les Lele estiment qu'il vient s'offrir de sa propre initiative : il se laisse tomber des arbres et au lieu de s'enfuir, il s'enroule sur lui-même, immobile. Le chasseur n'a qu'à attendre qu'il se déroule et sorte la tête pour le tuer (Douglas 1957 : 50). C'est bien en termes de sacrifice qu'il faut interpréter la mort et la consommation alimentaire de cet animal. Un sacrifice quelque peu sacrilège. Car c'est le représentant même, quasi humain, des esprits qui est dépecé. Les Lele éprouvent une certaine honte à manger ce "chef". Le rituel est conduit par les membres de l'association cultuelle des hommespangolins qui ont prouvé leur fécondité en mettant au monde un garçon et une fille ; leur haut statut social répond à des exigences spécifiques que nous analyserons plus loin. Ils ont seuls le droit de consommer la chair de 1 'animal dont le rôtissage s'effectue en secret. Les parties non comestibles (les écailles et les os) sont livrés aux chiens. La langue, le cou, la côte et l'estomac sont enterrés sous un palmier dont le vin sera réservé ultérieurement aux membres de la société des "géniteurs" (Douglas 1975 : 43).

La capture du pangolin, son dépeçage quasi sacrificiel constituent le coeur de la vie magico-religieuse des Lele qui ignorent toute forme du culte des ancêtres. Les règles symboliques que nous venons d'esquisser expliquent pourquoi les Lele n'accordent aucun intérêt à la domestication des animaux, pas plus du point de vue rituel que du point de vue socio-économique. Le besoin de viande et le souci de disposer d'une "monnaie" pour assurer la circulation des femmes entre les clans n'a jamais conduit les Lele à élever des chèvres ou des cochons, contrairement à leurs voisins méridionaux (Douglas 1954 : 5). Toute nourriture carnée vient de la forêt. Les Lele vont jusqu'au bout de leur propre logique classificatoire. A leurs yeux, la chair de la chèvre ou du 
cochon domestiques participe de la catégorie hama; elle soulève les coeurs comme les excréments ou le pus, "pour la sipmle raison qu'ils sont élevés au village" (Douglas 1954 : 5). La même attitude de refus $s$ 'applique aux poules, mais de manière moins radicale; les hommes ne se privent pas d'en manger, alors que les femmes s'en abstiennent complètement car elles seraient plus sensibles que leurs compagnons masculins à la "pollution" de cette chair immonde (ibid.). En aucun cas 1a viande d'un cochon domestique ne servira de substitut à celle d'un animal sauvage. Mary Douglas fut fort étonnée de constater au début de son séjour qu'une carcasse de porc avait été découpée et transportée quelques miles plus loin pour être vendue aux tribus voisines, alors que plusieurs chasses avaient été infructueuses et que la faim se faisait sentir ( $i b i d$.$) .$

La raison profonde de l'exclusion des chèvres et des porcs du régime alimentaire comme de la scène rituelle est que la place des animaux n'est pas parmi les hommes. Le chien, qui les accompagne à 1a chasse, est lui-même hama, dégoûtant, comme le rat qui hante les greniers ou les maisons de $1^{\prime}$ homme. Ce partage topologique est d'une rigueur implacable.

L'attituảe cérémoniezze des Tetela-Hamba face au monde de la forêt 1

A quelques centaines de kilomètres vers l'est, les Hamba manifestent la même indifférence à l'égard du traitement rituel de l'animal domestique. Mais ils sont loin de dédaigner l'élevage de la chèvre. Bien au contraire, celle-ci est par excellence l'unité de compte dans toutes les transactions socio-économiques, et singulièrement dans la circulation des femmes. La chèvre figure tradtionnellement parmi les valeurs matrimoniales, au même titre que les "monnaies" de fer et de cuivre, alors que les Lele attribuent cette fonction aux tissus de raphia. La chèvre n'en est pas moins absente, comme chez les Lele, de la scène sacrificielle. La structure de parenté (patrilinéaire et non plus matrilinéaire) diffère considérablement de celle des Lele, comme la culture. Les Hamba vivent à la lisière de la grande forêt ; ils appartiennent, comme leurs proches parents, les Tetela de la savane

1. Notes d'enquêtes personnelles. 
méridionale, au groupe linguisitique mongo qui peuple l'ensemble de la cuvette congolaise. Agriculteurs, les Hamba sont plus particulièrement passionnés par la chasse. Contrairement aux Lele cependant, celle-ci n'a pas engendré chez les Hamba d'activité magio-religieuse. Elle n'implique aucune tentative d'entrer en contact avec les mystérieux esprits edimu. Les Hamba et les Tetela se les représentent comme des êtres radicalement différents des hommes, de la même façon que les Lele décrivent les esprits mingehe. Ils hantent les sources comme les villages abandonnés. En tout état de cause, leur contact est dangereux et aucun culte positif ne permet de se les concilier. Chez les Tetela les rites de guérison pratiqués par les wetski-okunda (à la fois devins et guérisseurs) sont essentiellement fondés sur l'exorcisme. Le carrefour est le lieu privilégié de cette expulsion. On pourrait le définir comme un anti-autel, à la limite du monde des hommes et des esprits. Une poule vivante y est parfois abandonnée après avoir été frottée contre le corps du patient. La poule est par ailleurs abondamment consommée. Elle est le cadeau de bienvenue par excellence, offerte à un hôte de marque. Cet animal n'intervient que dans un seul rite positif. I1 est tout entier d'ordre magique. Lorsque les guerriers partaient jadis au combat, les wetshi préparaient un repas (oseto) dont une poule était l'ingrédient principal ; les participants 1'absorbaient avec avidité et précipitation. Ils étaient alors censés s'envoler au combat avec la rapidité de cet animal. Le même repas est effectué -- mais cette fois avec la chair d'un écureuil, animal réputé pour son caractère éveillé -- pour fortifier l'enfant à naître, durant le septième mois de la grossesse. Les futurs parents ainsi que les proches y participent. Lorsque $\mathrm{j}$ 'assistai à ce rite chez les Hamba, 1'organisateur refusa un lémurien que les enfants venaient de chasser sous prétexte que l'enfant risquerait de naître avec les gros yeux endormis de cet animal et d'être balourd. Qu'il s'agisse de la poule ou de l'écureil, le jugement qui préside à leur choix est évidemment d'ordre métaphorique.

Les wetshi okunda ne pratiquent aucun autre rite de fécondité. Lorsqu'ils vont prélever des herbes ou des racines en forêt pour confectionner les remèdes, ils ne communiquent ni avec les dangereux esprits edimu, ni avec les animaux. L'efficacité des plantes dépend de Dieu qui est souvent invoqué au moment de la cueillette. Cependant 
les wetshi okunda sont en rapport avec un esprit de la forêt spécifique, odyenge. Cette mystérieuse entité prend brusquement possession du fils ou de la fille d'un wetshi défunt, dans une espèce d'élection violente. L'élu rompt toute communication avec les hommes et s'enfuit en forêt. Il y découvre un sac contenant de 1'argile blanche qu'il utilisera pour les purifications. Un sacrifice exceptionnel, celui du chien, a lieu lors de sa réintégration au village. Cet acte s'inscrit dans un ensemble de rites qui portent la marque de la transgression. Le candidat s'approche de sa soeur (ou de son frère s'il s'agit d'une femme) dont il touche furtivement le sexe, tandis que les autres membres de la confrérie entonnent des chants obscènes. Un chien est mis à mort et le candidat en absorbe le coeur afin d'acquérir, dit-on, le flair de cet animal dans la chasse aux sorciers. On l'initiera alors au maniement de la calebasse divinatoire. L'acquisition du pouvoir de voyance se situe donc dans une zone ambiguë qui articule l'espace de la forêt et l'espace du village.

Chez les Hamba, une institution nouvelle, les "mâttres de la forêt" (nkum'okunda) s'est attribuée une part importante du pouvoir dévolu traditionnellement aux chef de lignage. Cette confrérie masculine fermée pratique des rites secrets dans une enceinte construite loin du village et interdite aux femmes comme aux non-initiés. Les nkum'okunda s'attribuent collectivement des tributs de chasse et imposent à cette activité un certain nombre d'interdits, variables d'un groupe à 1 'autre. D'une manière générale, la capture de quatre animaux est théoriquement prohibée : au léopard et à l'aigle, symboles d'autorité, s'ajoutent deux fourmiliers, le pangolin et l'oryctérope. Lorsqu'un léopard ou un pangolin ont été capturés, ils sont l'objet d'un traitement cérémoniel. Le chasseur doit acquitter des amendes importantes pour avoir enfreint les "interdits de chasse".

J'ai assisté à l'entrée au village d'un léopard mort chez les Djumbusanga. Deux hommes apportent le félin attaché par les pattes à une perche portée sur l'épaule. Ils vont de maison en maison, exigeant de chaque chef de famille le cadeau traditionnel de bienvenue (wema) auquel les chefs de lignage ont droit, puis ils suspendent l'animal à un bâti. Les mâ̂tres de la forêt se rassemblent ; ils sont accueillis par le chef du segment de lignage auquel appartient le chasseur. Ils lui imposent une amende parce qu'à leur arrivée l'animal était toujours 
suspendu à la perche alors qu'il aurait dû être couché sur un lit. Le chasseur acquitte le droit de "percer" 1'animal (otunguna wa nkoy). La viande est partagée entre le chef du lignage, les mâ̂tres de la forêt, le chasseur et le père. Quelques jours plus tard, ce dernier invite la confrérie et les chefs de lignage à manger ensemble la tête du léopard, non sans s'acquitter d'un nouveau paiement substantiel.

Cet interdit de chasse ne porte donc pas sur la consommation alimentaire, mais bien sur le meurtre de l'animal. Le traitement cérémoniel de 1 'animal et le flux de biens rares qui l'accompagne sont la conséquence directe de la mort accidentelle du léopard, représentant du pouvoir des Aînés dans le monde animal. Chez les Tetela de la savane, le chef de lignage, aîné de la branche aînée, doit accomplir un grand potlatch lors de son investiture, avant de pouvoir danser la danse rituelle au cours de laquelle il surgit de la forêt, semblable à un léopard : le corps moucheté de kaolin, il porte sur le dos la peau de l'animal et lance alternativement le bras gauche et le bras droit vers le haut dans un mouvement vigoureux qui exprime sa vitalité (de Heusch 1954). Les tambourinaires et la foule 1 'escortent joyeusement jusqu'au village. Ses épouses et ses proches parents 1 'accompagnent. Tous ont le corps aspergé de taches blanches évoquant le pelage de l'animal et certains portent en collier une ou plusieurs canines. L'assimilation du chef de lignage au félin est si forte chez les Tetela qu'il s'abstient d'en consommer la chair car "le léopard ne mange pas le léopard". Ses plus proches parents en ligne patrilinéaire appartiennent comme Iui au segment (1ittéralement "ventre") du léopard. Le flux de richesses que je viens d'évoquer à propos de la danse du léopard s'impose encore lors du deuil d'un chef de lignage. On se demandera évidemment si l'accueil solennel du léopard pris au piège n'est pas, chez les Hamba, un rituel funéraire équivalent.

Qu'en est-il du pangolin ? Sa chair est tout entière réservée aux maîtres de la forêt. Mais une règle inverse prévaut dans certaines régions : l'animal est mangé à l'intérieur de l'enceinte, en forêt, si le chasseur est lui-même un initié, sinon la viande est abandonnée aux femmes et aux hommes. qui n'appartiennent pas à la confrérie. Si 1'oryctérope est aussi 1 'objet d'un interdit, c'est parce qu'il vit caché dans un terrier profond. En outre, ces deux fourmiliers ne mettent qu'un qu'un petit au monde à chaque portée, comme l'homme. Nous retrouvons 
donc chez les Hamba l'une des connotations métaphoriques au moins que possède le pangolin aux yeux des Lele. Mais les Hamba n'établissent, quant à eux, aucune connexion entre ces animaux interdits et les esprits. En outre, c'est un grand pangolin (manis gigantea), et non l'espèce arboricole, que $j^{\prime}$ 'ai vu les Hamba traiter cérémoniellement.

Si le petit pangolin est seul l'objet d'un rite quasi sacrificiel chez les Lele, on observera cependant que 1 'espèce géante se retrouve avec l'oryctérope dans la liste des animaux-esprits. Mais ces deux fourmiliers n'y occupent qu'une place secondaire: seules les femmes enceintes s'abstiennent de manger le manis gigantea et l'oryctérope est 1 'objet d'un interdit alimentaire réduit (Douglas 1957 : 40).

La différence d'attitude des Lele et des Hamba vis-à-vis des animaux interdits saute aux yeux. Les seconds se bornent à les traiter avec le respect d̂̂ aux chefs, sans que ce cérémonialisme n'influence la fécondité des femmes ou le succès de la chasse. En formalisant que1que peu la pensée hamba, qui ne s'embarasse pas d'un appareil classificatoire aussi compliqué que la taxinomie lele, on peut dire que le chef est 1 'homologue du léopard dans le monde des hommes, comme le pangolin, voire l'oryctérope, sont les représentants du monde hưain en forêt. Mais les Hamba ne tentent aucune médiation magico-religieuse entre nature et culture. Les maîtres de la forêt se contentent d'imiter joyeusement le comportement des animaux sauvages dans les danses de divertissement, sur un mode ludique.

L'association des maîtres de la forêt, qui s'approprie tributs de chasse et animaux interdits, est de nature politique, et non religieuse, contrairement à la confrérie des hommes-pangolins chez les Lele. Les objets et les pièges révélés aux nouveaux membres dans l'enceinte initiatique constituent autant de signes secrets du pouvoir. Ils s'inscrivent dans un ensemble symbolique vide. Les mâ̂tres de la forêt ne dispensent aucun enseignement mythique, n'engagent aucun dialogue avec les esprits errants de la forêt, dont la nature même deumeure énigmatique.

La pauvreté des activités strictement rituelles est tout aussi déconcertante à l'intérieur du lignage. Ni les Tetela, ni les Hamba n'adressent prières, offrandes ou sacrifices à leurs ancêtres. Les chefs de lignage soutiennent leur prestige par la générosité et non par des moyens mystiques. Cette' société, où la compétition pour les 
femmes, le désir de se procurer les biens de prestige, la rivalité des aînés et des cadets sont exacerbés, est hantée par la sorcellerie. Nulle institution religieuse ne s'est cristallisée autour des animaux du village ou de la forêt pour renforcer la cohésion sociale.

Ce bilan, négatif pour notre enquête comparée, se traduit notamment par deux différences significatives par rapport aux Lele. Symbole du pouvoir, et non de la sorcellerie, le léopard est traité comme le pangolin. Parmi les manidés, l'espèce privilégiée (manis gigantea) diffère elle-même de celle qui retient l'attention des Lele (manis tricuspis). Nous pouvons en apprendre davantage à ce sujet en interrogeant une autre société de la forêt équatoriale : les Lega du Maniéma. Interdit majeur de chasse, comme chez les Hamba, le grand pangolin se voit attribuer cette fois un véritable traitement sacrificiel.

Petit et grand pangolin (Lele et Lega)

Le parti-pris des Lega inverse celui des Lele : seul le grand pangolin (manis gigantea) est l'objet d'un traitement rituel. Les Lega établissent une hiérarchie entre les deux espèces. Le petit pangolin soulève peu d'intérêt ; il n'est en effet que le frère cadet du grand pangolin. En revanche, le "frère aîné" est respecté, protégé par un interdit de chasse strict. Lorsqu'on le trouve mort en forêt, il appartient tout entier à une association fermée, détentrice de l'autorité (1e bwami), comparable à certains égards à l'association des mâ̂tres de la forêt chez les Hamba. Commentons la description de Daniel Biebuyck.

Le grand pangolin ( $i k a g a)$ est aux yeux des Lega un héros culture1. C'est lui qui enseigna aux hommes l'art de construire la maison. Ses écailles superposées évoquent les tuiles de feuilles dont les Lega recouvrent le toit des huttes (Biebuyck 1973 : 224). Les détenteurs du grade supérieur (kindi) dans le bwami sont associés à cet animal bénéfique car leur fonction sociale est de "réunir" les gens, de maintenir la cohésion du groupe. Le dépeçage et le partage du grand pangolin font intervenir plusieurs lignages patrilinéaires apparentés. Un nombre impressionnant de personnes en bénéficient. Un neveu utérin du lignage organisateur est chargé de commencer les opérations. II enlève les écailles qui viendront s'ajouter aux objéts rituels détenus par les dignitaires de haut grade. Ceux-ci en jettent quelques-unes 
sur le toit de la case, sans doute pour confirmer leur association symbolique avec la maison. Assisté d'un autre neveu utérin, le premier officiant égorge ensuite un bouc qui est grillé tout entier dans le feu. Cet animal domestique sera partagé selon des règles assez semblables à celles qui président à la répartition de la chair du pangolin entre les initiés des différents lignages présents (Biebuyck 1953).

Les neveux utérins reçoivent obligatoirement les reins. Leur participation mérite l'attention. Un proverbe assimile en effet le grand pangolin à l'oncle maternel. Or la parenté maternelle est un des éléments essentiels de l'espace social qui se compose d'un réseau extrêmement étendu de type omaha (Biebuyck 1953) ${ }^{2}$. On comprend mieux ainsi cette figure proverbiale: "Pangolin, tu es mon oncle maternel qui étend les terriers au loin" (Biebuyck 1953: 910). Par le truchement de la relation avunculaire, c'est toute la construction sociale que symbolise ce fourmilier qui creuse des tunnels profonds dans le sol. La fonction du bouc qui accompagne le pangolin dans ce rituel n'est malheureusement 1'objet d'aucun commentaire. Après le repas, tous les participants vont obligatoirement se baigner pour se purifier car ils viennent d'enfreindre un très grand interdit (mueko) (Biebuyck 1953 : 924).

Tout se passe comme si le groupe venait de dépecer la parenté tout entière pour mieux la renforcer. Ce qu'ils ont accompli, à vrai dire, n'est autre que l'équivalent d'un repas cannibale, le sacrifice du héros civilisateur. La comparaison avec 1 'attitude honteuse des Lele, lorsqu'ils font rôtir la chair du petit pangolin, ce "chef" respecté, s'impose. Le rite du grand pangolin chez les Lega traduit sur un plan sociologique la fonction religieuse du petit pangolin chez les Lele. C'est précisément ce glissement symbolique qui explique le passage d'une espèce à l'autre.

Les Lele $n^{\prime}$ assimilent nullement le petit pangolin (manis tricuspis) à un parent. Les clans matrimoniaux lele sont dispersés et n'ont aucune cohésion sociale, à l'inverse des lignages patrilinéaires lega qui

2. Les Lega maintiennent des relations sociales étroites avec les lignages patrilinéaires des oncles maternels des parents et des quatre grands-parents. 
constituent des unités résidentielles. La fonction logique du petit pangolin dans le premier cas est d'un autre ordre que celle du grand pangolin dans le second. Pour s'en rendre compte, il suffit d'examiner les règles d'accession à la société cultuelle des hommes-pangolin. Tout candidat doit pouvoir exciper des qualités suivantes : i1 doit avoir donné un garçon et une fille à la même épouse, membre d'un clan fondateur du village (permettant ainsi au système matrilinéaire de se reproduire) ; en outre, il doit appartenir lui-même, comme son père, à 1 'un des clans fondateurs. Mary Douglas aperçoit fort bien que ces règles encouragent les intermariages entre ces clans afin de combattre 1 'hémorragie démographique dont le village est sans cesse menacé (Douglas 1963 : 209). Le petit pangolin contribue donc à la synthèse sociologique de deux institutions complémentaires et antagonistes : le village, unité politique permanente, et le clan matrilinéaire dispersé, émietté en sections locales dépourvues d'institutions propres. Cettefonction unificatrice est centripète : elle affirme la nécessité de maintenir au sein du village un réseau d'alliances endogamiques entre les mêmes clans. Le petit pangolin fait revenir au village fondé par leurs ancêtres des hommes et des femmes nés au dehors en vertu du mariage virilocal ${ }^{3}$. Dans la société patrilinéaire lega, le petit pangolin assume une fonction inverse : il rassemble dans une vaste communauté rituelle un réseau de villages, solidement définis par leur unité lignagère. Dans les deux cas, cependant, le pangolin est un puissant facteur de cohésion sociale.

Une différence plus importante peut être dégagée. La taille de I'animal est un élément pertinent là où $I^{\prime}$ â̂nesse est valorisée : dans 1a pensée lega, le grand pangolin est le frère aîné de 1'autre ; il signifie la construction sociale tout entière : l'ordre hiérarchique à 1 'intérieur du lignage aussi bien que le réseau étendu des parents maternels. On retrouve les mêmes caractéristiques sociologiques fondamentales chez les Hamba qui privilégient le grand pangolin : 1'aînesse détermine la hiérarchie des lignages et la relation avunculaire est 1 'une des pierres angulaires complémentaires de la société patrilinéaire. En revanche, l'â̂nesse n'est pas un principe organisateur de

3. Je m'écarte ici légèrement de 1'interprétation proposée par Mary Douglas dans Implicit Meaning (pp. 297-298). 
la société lele; la hiérarchie des deux pangolins se renverse pour des raisons qui ne relèvent plus de 1'ordre sociologique, mais d'une symbolique cosmique. manis tricuspis est 1a seule espèce arboricole du genre manidé. A ce titre le petit pangolin se situe au carrefour des trois ordres vivants : comme le note Mary Douglas elle-même, il est le seul mammifère quadrupède ressemblant à. la fois à un poisson et à un oiseau (Douglas 1975: 46, note 13). La richesse cosmogonique du petit pangolin surpasse évidemment, de ce point de vue, celle de son homologue géant.

Le même point de vue explique encore la prééminence que manis tricuspis détient sur manis gigantea dans le symbolisme royal kuba où il figure parmi les animaux "nobles dévolus au souverain" (Vansina 1964 : 109). La fonction rituelle de la royauté sacrée n'est-elle pas de contrôler 1'ensemble des forces naturelles ? Le roi sacré est à la fois au coeur de 1 'ordre humain et en dehors de celui-ci, au coeur de la nature. Comme le pangolin. Lorsqu'elie eut pris connaissance de mon livre Le roi ivre, où je commençais à exposer les fondements symboliques de la royauté sacrée en Afrique centrale -- et particulièrement chez les Kuba -- Mary Douglas admit qu'elle regrettait de n'avoir jamais eu accès à la connaissance ésotérique des hommes-pangolins et de ne pas avoir analysé un certain nombre de "contes" qui lui apparaissent aujourd'hui susceptibles d'éclairer le symbolisme cosmogonique du pangolin dans lequel elle est prête à voir "le signe potentiel de 1 'union du ciel et de la terre" réalisée par les rois sacrés (Douglas $1975: \mathrm{X})$.

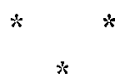

Le traitement rituel du pangolin chez les Lele et les:Lega nous a amené à poser la question du sacrifice par le biais des classifications opérant à l'intérieur du vaste monde des animaux sauvages. Que ceux-ci soient "bons à penser", Lévi-Strauss nous 1 'a appris il y a longtemps. Le pangolin est d'abord l'objet d'un interdit. Les Lele comme les Lega le mangent avec un mélange de cérémonialisme et de répugnance. Les seconds vont se purifier à la rivière après l'avoir consommé, conme s'ils avaient commis un acte sacrilège. Le pangolin n'a rien d'une espèce "totémique" évidemment et c'est en vain que 1 'on s'évertuerait à voir dans la manducation rituelle de cet animal interdit une confirmation 
de la thèse de Robertson Smith -- critiquée à juste tire par Hubert et Mauss -- selon laquelle une prétendue communion alimentaire ayant pour objet l'espèce "totémique" interdite, "sacrée", serait à l'origine du sacrifice.

Le rituel du pangolin ne s'accompagne pas moins d'une consommation alimentaire aux vertus mystiques. Les Lega resserrent leurs liens sociaux au cours de ce repas, tandis que les hommes-pangolins lele renforcent leurs relations privilégiées avec les sources mêmes de la fécondité par le truchement de l'animal interdit. Quelque chose de l'ordre du sacrifice pointe à l'horizon.

Mais la comparaison ne vaut que pour la phase finale, le repas. La phase initiale, la mise à mort fait défaut. L'animal, au contraire, est interdit et il vient s'offrir de son plein gré pour assurer la. communication avec le monde des esprits dans le schéma lele.

I1 serait faux de prétendre que la communication accidentelle et quasi sacrilège du pangolin occupe chez les Lele la place, ici vacante, du sacrifice des animaux domestiques, pratiqué par la majorité des sociétés bantoues, éleveurs de chèvres ou de boeufs. La victime volontaire de ce sacrifice particulier est un représentant des esprits de la nature. A ce titre, le petit pangolin est dans le système symbolique lele 1 'équivalent rịgoureux du roi sacré kuba, régulateur de 1 'ordre cosmique comme de 1 'ordre social. L'association du pangolin et de la royauté traverse toute l'Afrique bantoue car on la retrouve chez les Lovedu du Transvaal : la graisse de cet animal, capturé vivant, entre dans la composition de la médecine de pluie mise en oeuvre par la reine (Krige 1943: 274).

Le fait que les Lele associent le petit pangolin à la fécondité générale en le saluant du titre de "chef", lui conférant la puissance même que leurs voisins kuba attribuent au seul souverain, suggère que cette créature, décidément étrange, est susceptible de nous frayer un passage vers la plus singulière des institutions sacrificielles : la mise à mort rituelle des rois. 


\section{Bibliographie}

BIEBUYCK D.

1953 Répartition des droits du pangolin chez les Balega, Zaire, VII, 8, 899-934

1973 Lega Cuzture (Art, Initiation and Moral Philosophy among a Central African People), Berkeley-Los Angeles, London

DOUGLAS M.

1954 The Lele of Kasai, in D. Forde (éd.) African Worlds (Studies in the Cosmological Ideas and Social Values of African Peoples), Oxford University Press, London-New York-Toronto, $1-26$

1955 Social and Religious Symbolism of the Lele of Kasaj, Zaire, IX, 4, 385-402

1957 Animals in Lele Religious Symbolism, Africa, XXVII, I, 46-58

1963 The Lele of the Kasai, Oxford University Press

1975 Implicit Meaning (Essays in Anthropology), London-Boston

HEUSCH L. de

1954 Autorité et prestige dans la société tetela, Zärre, VIII, $10,1001-1027$

1971 Préface à Mary Douglas De Za souizlure (Essai sur les notions de pollution et de tabou), Paris, 7-20

KRIGE J. D. \& E. J.

1943 The Realm of the Rain-Queen (A Study of the Pattern of Lovedu Society), London-New York-Toronto

VANSINA J .

1964 Le royaume kuba, Musée Royal de l'Afrique Centrale, Tervuren 

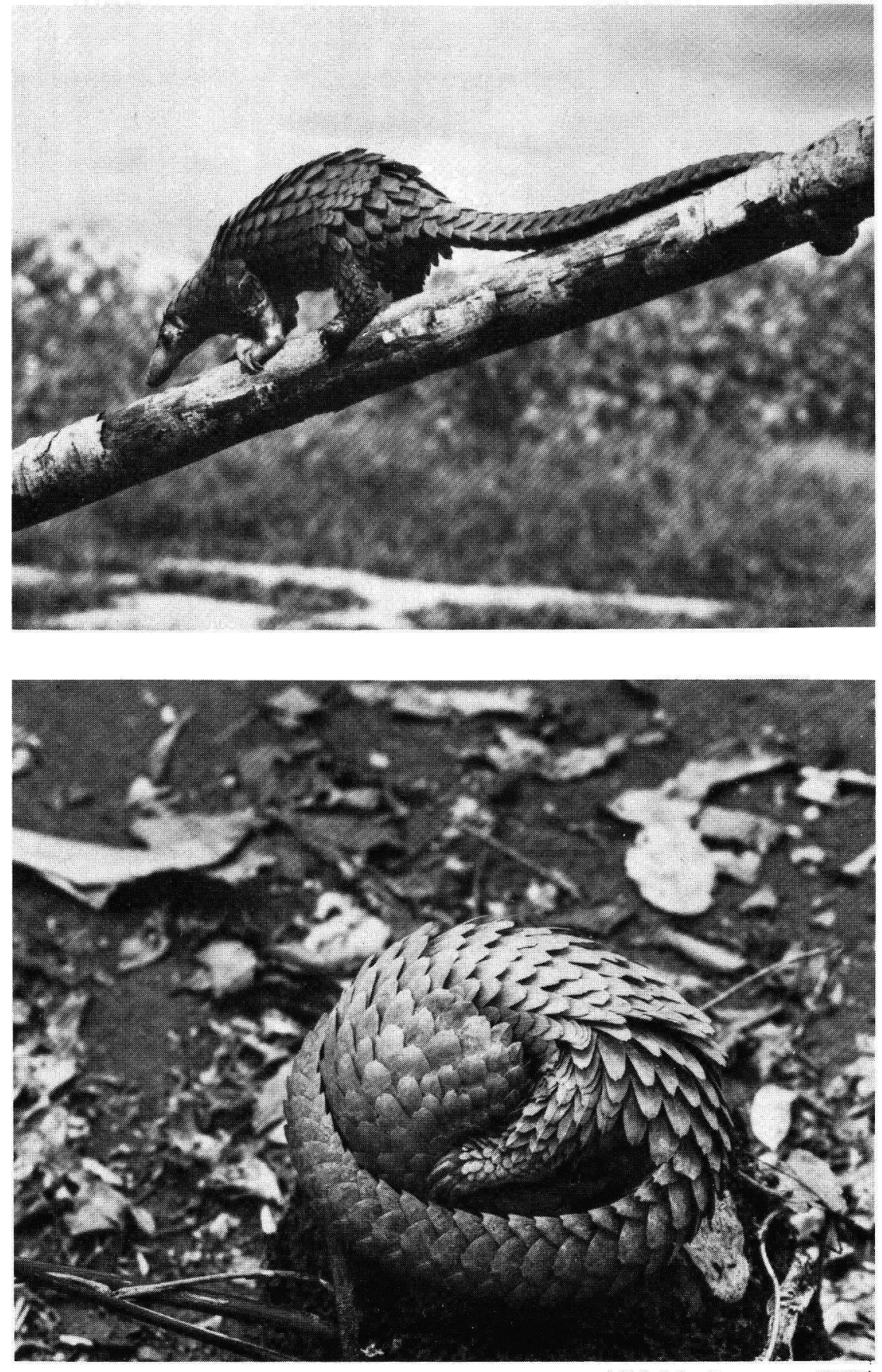

(Clichés Devese - CNRS)

Manis tricuspis (petit pangolin) 




(Cliché Devese - CNRS)

Manis tricuspis 